\title{
Using a Relativistic Electron Beam to Generate Warm Dense Matter for Equation of State Studies
}

M. J. Berninger

National Security Technologies, LLC, Los Alamos, NM 87544

T. J. T. Kwan, M. J. Schmitt

$X$-Computational Physics Division, Los Alamos National Laboratory, Los Alamos, NM 87545 


\section{Abstract}

Experimental equation-of-state (EOS) data are difficult to obtain for warm dense matter (WDM)ionized materials at near-solid densities and temperatures ranging from a few to tens of electron voltsdue to the difficulty in preparing suitable plasmas without significant density gradients and transient phenomena. We propose that the Dual Axis Radiographic Hydrodynamic Test (DARHT) facility can be used to create a temporally stationary and spatially uniform WDM. DARHT has an $18 \mathrm{MeV}$ electron beam with $2 \mathrm{kA}$ of current and a programmable pulse length of $20 \mathrm{~ns}$ to $200 \mathrm{~ns}$. This poster describes how Monte Carlo n-Particle (MCNP) radiation transport and LASNEX hydrodynamics codes were used to demonstrate that the DARHT beam is favorable for avoiding the problems that have hindered past attempts to constrain WDM properties.

In our concept, a $60 \mathrm{~ns}$ pulse of electrons is focused onto a small, cylindrical (1 mm diameter $\times 1 \mathrm{~mm}$ long) foam target, which is inside a stiff high-heat capacity tube that both confines the WDM and allows pressure measurements. In our model, the foam is made of $30 \%$ density Au and the tamper is a B4C tube. An MCNP model of the DARHT beam investigated electron collisions and the amount of energy deposited in the foam target. The MCNP data became the basis for a LASNEX source model, where the total energy was distributed over a $60 \mathrm{~ns}$ time-dependent linear ramp consistent with the DARHT pulse. We used LASNEX to calculate the evolution of the foam EOS properties during and after deposition. Besides indicating that a $\sim 3 \mathrm{eV}$ Au plasma can be achieved, LASNEX models also showed that the WDM generates a shock wave into the tamper whose speed can be measured using photonic Doppler velocimetry. EOS pressures can be identified to better than $10 \%$ precision. These pressures can be correlated to energy deposition with electron spectrometry in order to obtain the Au EOS. Radial uniformity in the DARHT beam was also investigated. To further obtain uniform radial energy deposition, MCNP calculations were carried out with radial beam filters. Results are presented. 


\section{Dense plasma EOS, what physics matters?}

- Our EOS models rely on a separation of thermodynamic quantities into three components: the cold curve, thermal ionic, and thermal electronic

$$
\begin{aligned}
& P(\rho, T)=P_{c}(\rho, T)+P_{e}(\rho, T)+P_{n}(\rho, T) \\
& E(\rho, T)=E_{c}(\rho, T)+E_{e}(\rho, T)+E_{n}(\rho, T) \\
& F(\rho, T)=F_{c}(\rho, T)+F_{e}(\rho, T)+F_{n}(\rho, T)
\end{aligned}
$$

- The lower pressure regime is dominated by the cold curve and thermal ionic components of the pressure and energy

This also happens to be where we have data.

- In the dense plasma regime where the temperature is $10 \mathrm{~s}$ to $100 \mathrm{~s}$ of $\mathrm{eV}$, the EOS is dominated by the thermal electronic part?

Do we calculate this correctly?

Very little data exist in this regime. 


\section{EOS of WDM is largely unexplored (both theory and data) and therefore has high uncertainty}

We construct EOS tables using the interpolation method:

$$
\begin{aligned}
& F_{\text {tot }}=F_{\text {cold }}+F_{\text {nuclear }}+F_{\text {elec-thermal }} \\
& \text { cold matter: }
\end{aligned}
$$

In WDM, we are in the interpolated regime between known physics limits. This is a very general issue in the field of EOS physics: we have developed accurate methods in extreme limits where very good approximations work, but not in the center.

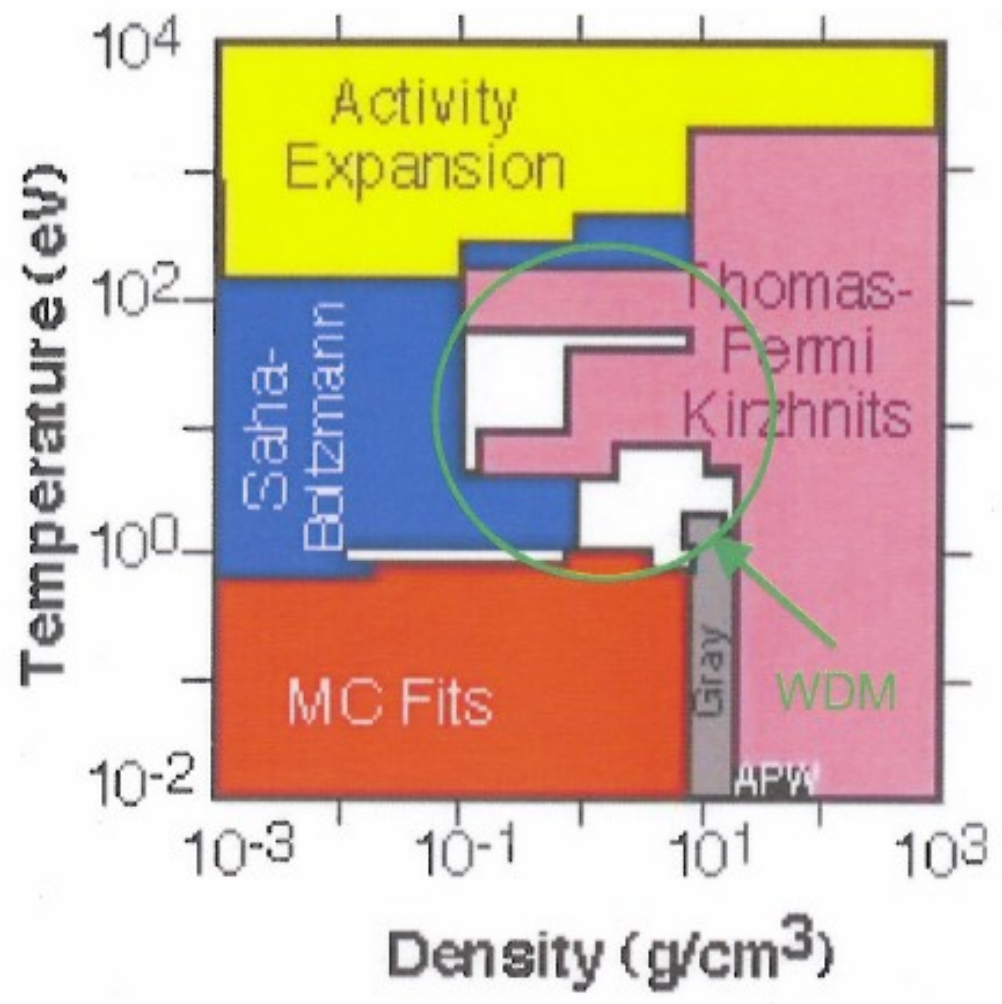




\section{A variety of attempts at obtaining data have been tried}

- In general, these attempts have either been unsuccessful or have required simulations to model the experiment

- We examined several different experimental approaches and evaluated each as to whether a complete EOS could be obtained, the range of parameter space that could be addressed, and the flexibility of the approach to address various materials

- The various approaches we considered were:

1. Laser-heated thin slab experiments

2. Isochorically heated targets

3. Ohmic heating of thin metal foils

4. Laser-driven shock experiments

5. Shock and release experiments, both flyer plate and laser-driven 


\section{WDM with different transient characteristics can be created by several energetic beams- the DARHT beam is the most suitable}

- Short pulse lasers

- Trident: $\lambda=1 \mu \mathrm{m} ; \mathrm{E}=100 \mathrm{~J} ; \tau=1$ ps

- Surface interaction with thin gold foil resulting in $10 \mathrm{~s} \mathrm{eV}$ plasma with rapid temporal evolution and steep spatial gradients

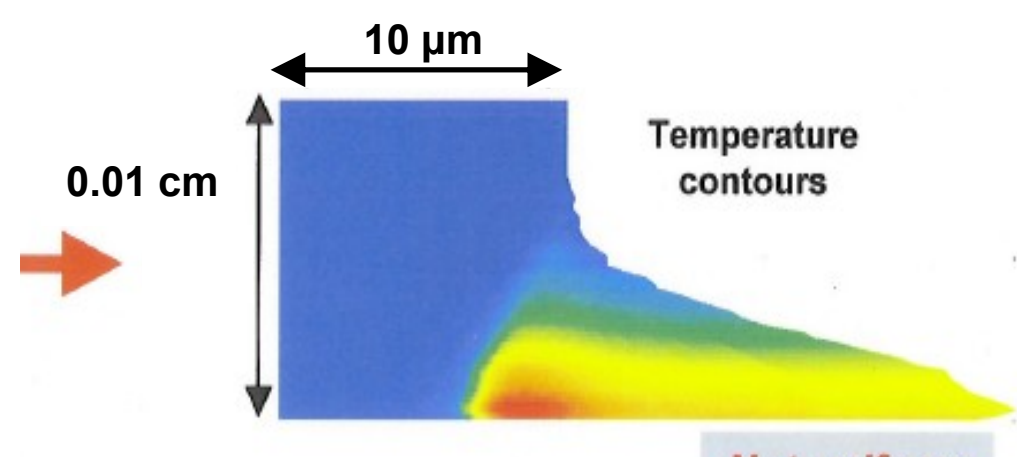

- Energetic ion beams

- LANSCE $800 \mathrm{MeV}$ proton beam $N_{p}=1 \times 10^{13} ; \tau=240 \mathrm{~ns}$

- Energetic deposition into a $\mathrm{Pb}$ thin wire results in about $1 \mathrm{eV}$ plasma with steep radial gradients and unfavorable temporal behavior

- Energetic electron beams

- DARHT-2 $18 \mathrm{MeV}$ electron beam; I = $1.8 \mathrm{kA}$ with a programmatic pulse length format from 20 ns to 200 ns

- For a 60 ns pulse, gold foam with $30 \%$ gold density can be converted to a $\sim 3 \mathrm{eV}$ plasma within a tamped configuration
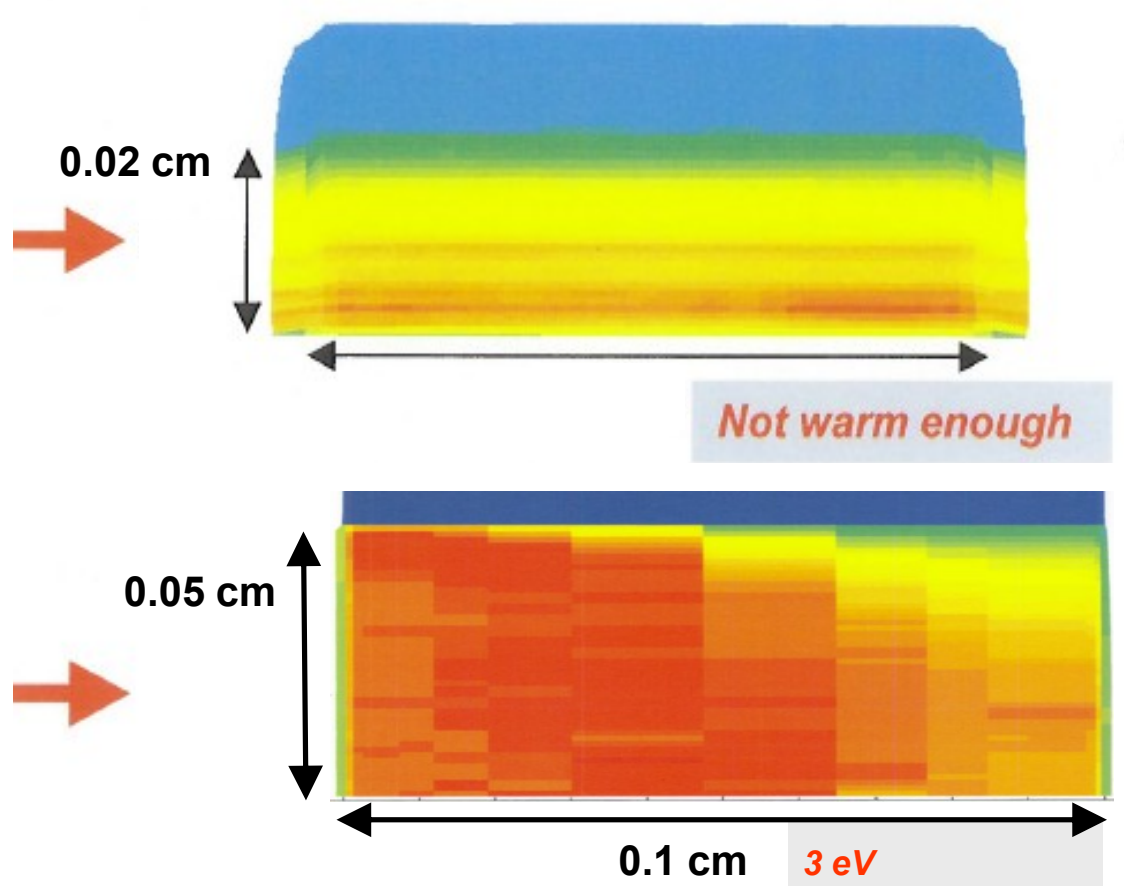


\section{Using the flexible pulse format of the $2^{\text {nd }}$ axis of the DARHT electron beam, gold foam WDM can be created with a range of temperatures $(1 \mathrm{eV}$ to $10 \mathrm{eV})$ with known density}

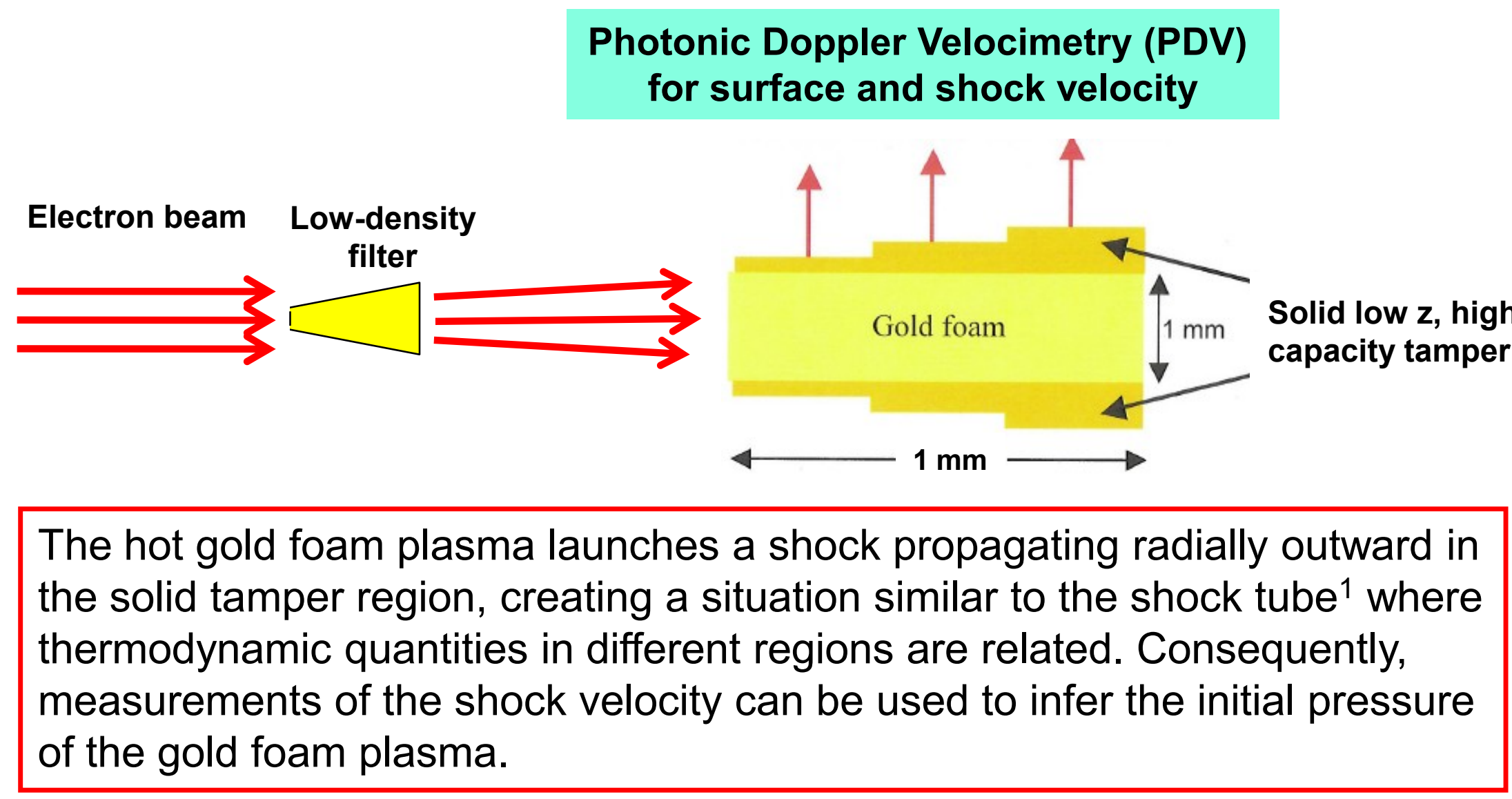

${ }^{1}$ Francis H. Harlow and Anthony A. Amsden, "Fluid Dynamics," Los Alamos Scientific Laboratory Monograph LA-4700, (June 1971). 


\section{DARHT beam properties}

- The DARHT beam parameters were combined in a detailed source model programmed in FORTRAN and compiled with MCNP5

- Energy, $T_{\text {beam }}=18 \mathrm{MeV}$

- Current, $I=1.8 \mathrm{kA}$

- Gaussian spatial profile: $\sigma_{x}, \sigma_{y}=0.032 \mathrm{~cm}$

- Data show that the DARHT beam has an emittance of $\varepsilon_{n}=1000 \pi \pm 500 \pi \mathrm{mm}$-mrad; divergence angle $\sigma_{\theta}=2.5$ degrees

- The emittance plot to the right shows that the location and divergence of the electrons in the beam forms an elliptical 2D Gaussian distribution.

- The nominal area of the ellipse is $\varepsilon n \propto \pi \sigma x \sigma \theta$; given $\sigma x, \sigma \theta$ is easily found

- The divergence is greatest in the center of the spot

2D spatial Gaussian source
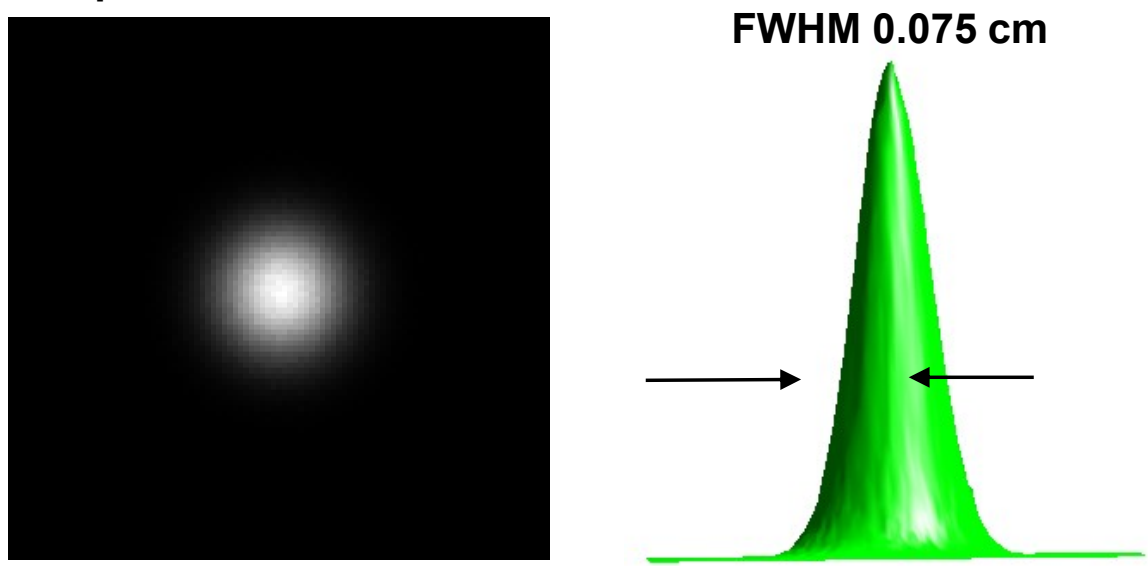

Emittance $\varepsilon_{n}=1000 \pi$

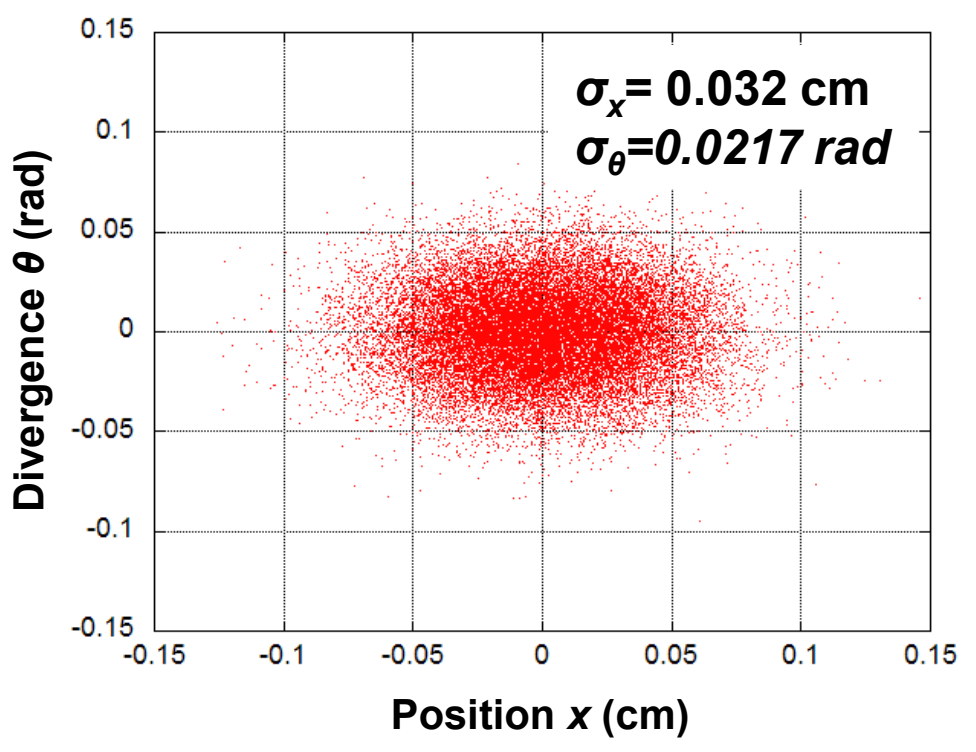




\section{Features of the WDM model in MCNP5}

Monte Carlo N-Particle ${ }^{2}$ v. 5 (MCNP5) was used to calculate the transport of energetic electrons and $\mathrm{x}$-ray radiation in a WDM target

MCNP5 calculates energy deposited due to electron multiple scattering with the filter, collimator, and the foam target.

The calculation used a detailed electron beam model with Gaussian spatial distribution and emittance

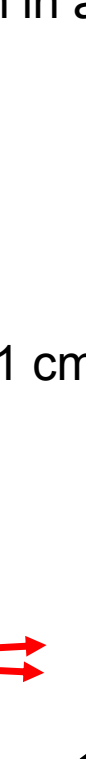

Au filter mask $\rho=0.23 \mathrm{~g} / \mathrm{cm}^{3}$

MCNP shows the temperature of the low density Au foam filter reaches $1000 \mathrm{~K}$; well below the melting point $1340 \mathrm{~K}$.

Filter materials with different $Z$ produced very similar interactions in the foam target. The filter material, Au, was chosen to prevent contamination.

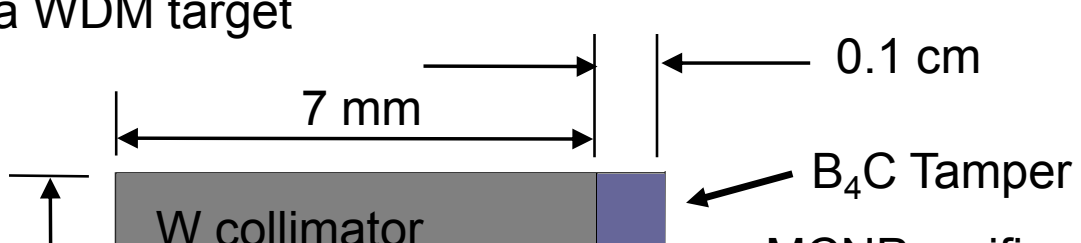

MCNP verifies only a small proportion of the electron scatter into the tamper through the collimator due to the small range $4.6 \mathrm{~mm}$ of $18 \mathrm{MeV}$ electrons in $\mathrm{W}$
0.1

$0.1 \mathrm{~cm}$

$\Theta \sim 1$ degree
Au Foam

$\rho=6.4 \mathrm{~g} / \mathrm{cm}^{3}$

Radiation dose was calculated with high spatial resolution in foam and tamper 


\section{MCNP calculations with radial beam filters}

Due to the Gaussian spatial distribution in the DARHT beam, it is necessary to use a filter mask to spatially redistribute the energy deposited in the foam. The figures below show the results of placing a Au foam conical frustum $\sim 7 \mathrm{~mm}$ in front of the target (frustum: $4 \mathrm{~mm}$ long, $\rho_{\mathrm{Au}}=0.23 \mathrm{~g} / \mathrm{cc}$; angle $\theta \sim 1$ degree)

Au foam target
$\rho_{\mathrm{Au}}=6.4 \mathrm{~g} / \mathrm{cc}$

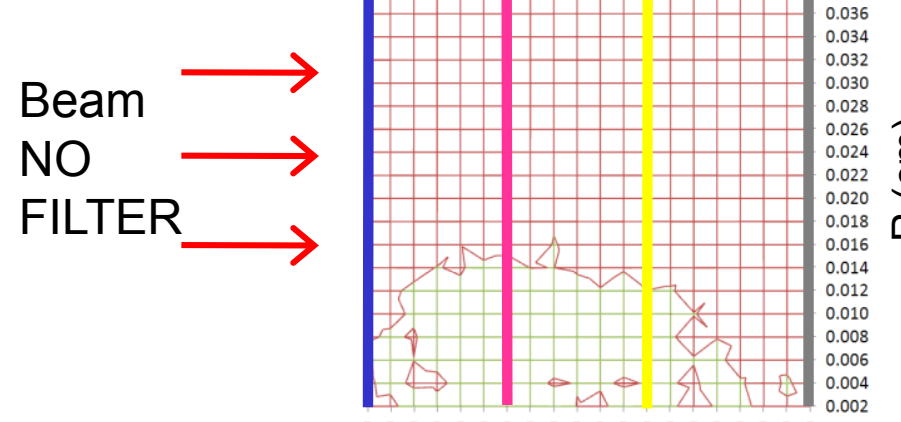

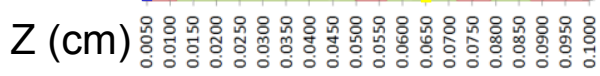

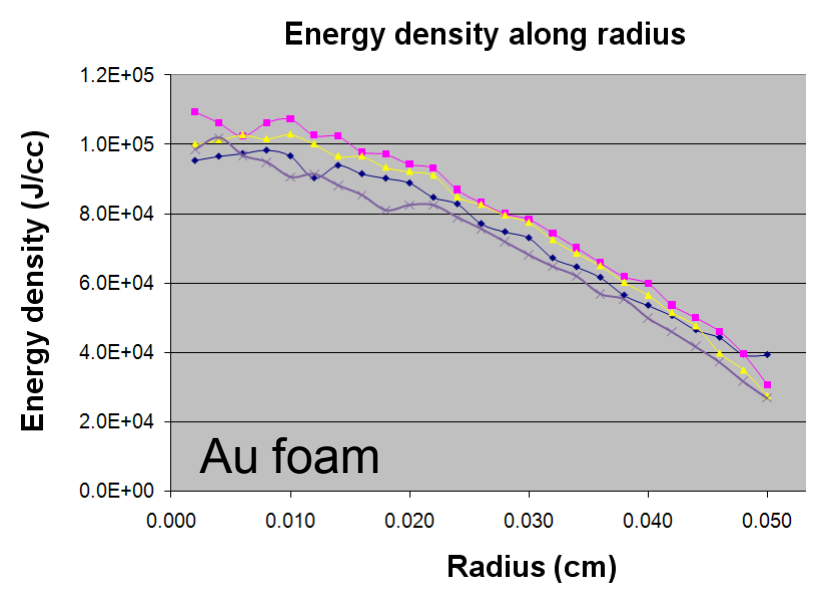

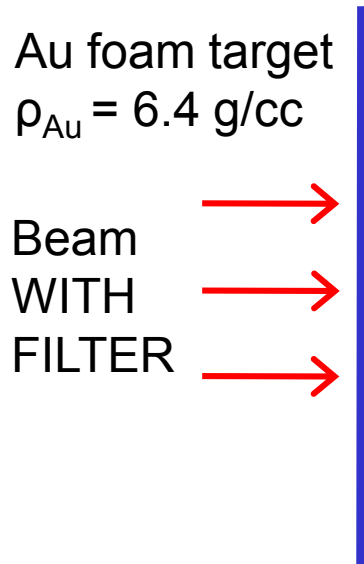
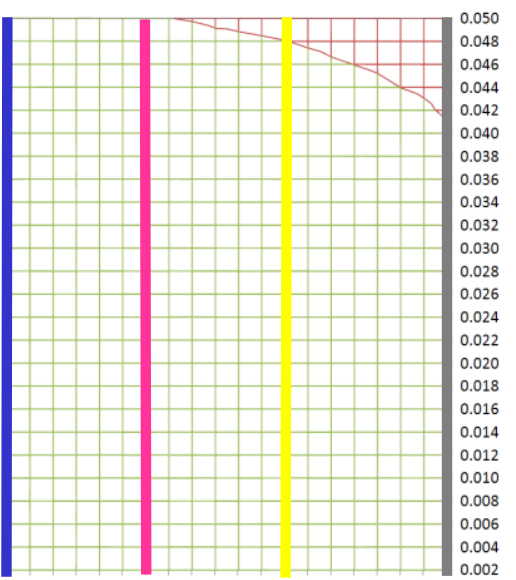

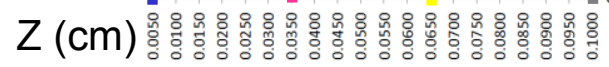

An

unfiltered

beam

Z (cm)

results in

energy

density

gradients

that can

obscure the

pressure

measure-

ment
Energy density along radius

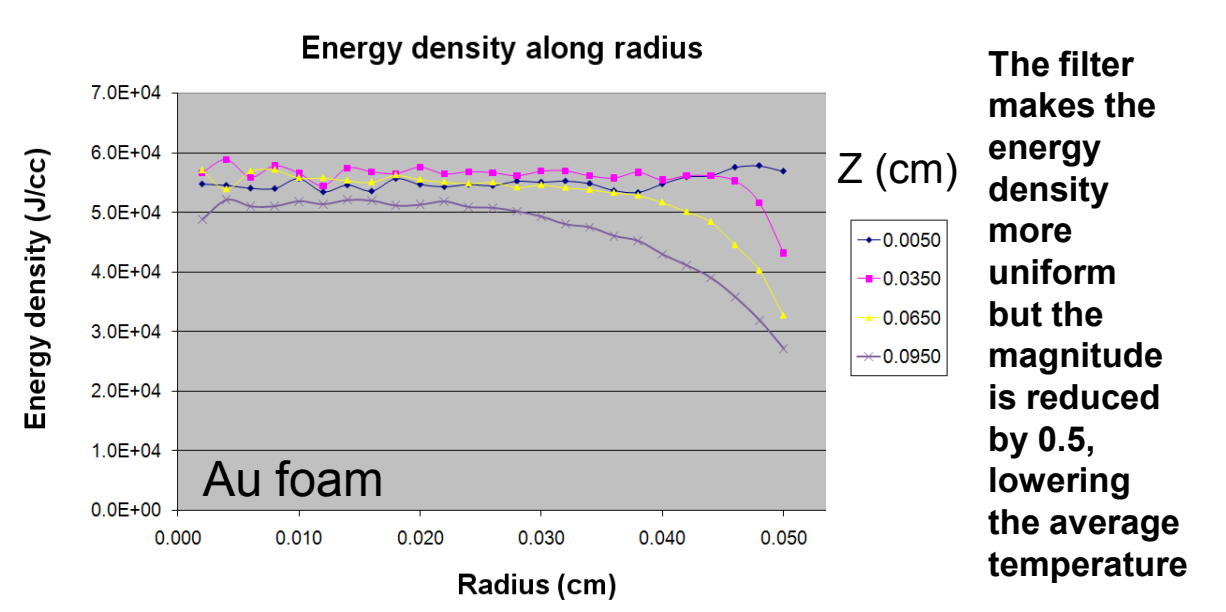

$E_{\text {dens }}(\mathrm{J} / \mathrm{cc})$

$-1.00 E+04-6.00 E+04$
$2.00 E+04-4.00 E+04$ $00.00 E+00-2.00 E+04$ 


\section{Beam target interaction: angle dependencies}

-When designing the Au foam target, we must anticipate electron beam deflections due to

- Beam divergence

- Electron scattering from the filter mask

- Electron scattering in the target

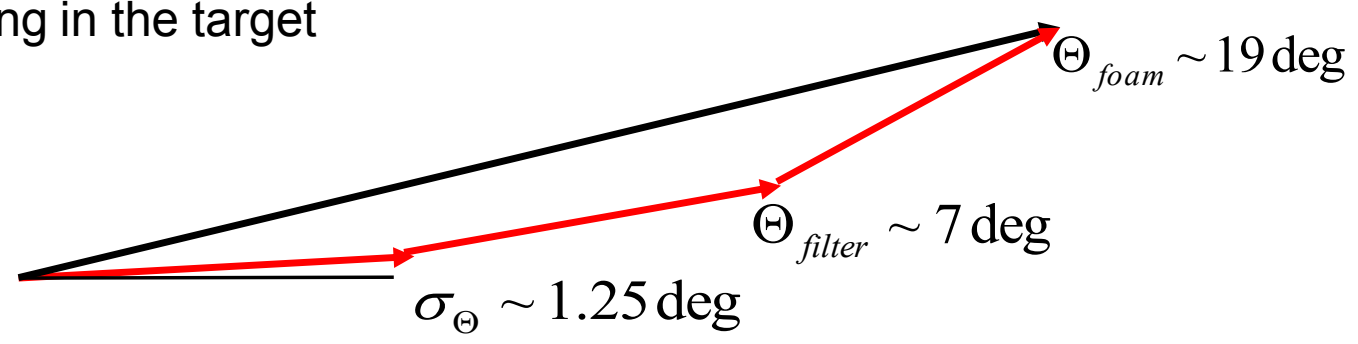

- We made a crude approximation to predict the net electron scattering deflection angle, $\Theta$, that combines all three statistical quantities

$$
\Theta \propto \Theta_{\text {foam }}(T, Z, \rho)+\Theta_{\text {filter }}\left(T, Z^{\prime}, \rho^{\prime}\right)+\sigma_{x^{\prime}}\left(\varepsilon_{n}, \sigma_{\text {spot }}\right),
$$

Where $\sigma_{x^{\prime}}\left(\varepsilon_{n}, \sigma_{\text {spot }}\right)$ is the divergence of the beam that depends on the beam emittance $\varepsilon_{n}$ and beam spot size $\sigma_{\text {spot }}$. The angles $\Theta_{\text {foam }}(T, Z, \rho)$, and $\Theta_{\text {fitter }}\left(T, Z^{\prime}, \rho^{\prime}\right)$, are the cumulative deflections of the electron beam due to multiple scattering in the foam filter and the foam target, respectively. Here, $Z$ is the atomic number, $T$ is the beam kinetic energy and $\rho$ is areal density; unprimed quantities refer to the foam target and primed quantities to the filter mask.

- In the foam target system, the net scattering deflection, was $\sim 20-30$ degrees. As a "rule of thumb," this scattering angle was dominated by $\Theta_{\text {foam }}(T, Z, \rho)$, which was typically $\sim 19$ degrees.

- The filter deflection $\Theta_{\text {fitter }}\left(T, Z^{\prime}, \rho^{\prime}\right)$, half the magnitude to the foam, remained relatively constant regardless of material $Z$, density, or thickness. The reason is that the foam parameters had to be adjusted to compensate for the target foam in order to maintain a uniform energy density distribution. 


\section{Effects of beam emittance, $\varepsilon_{n}$}

- For a constant spot radius, $\sigma_{x}$, varying $\varepsilon_{n}$ changes the divergence angle of the beam. Our calculations used $\varepsilon_{n}=1000 \pi \mathrm{mm}$-mrad; with $\sigma_{x}=0.032 \mathrm{~cm}$ the divergence angle is $\sigma_{\theta}=2.5$ degrees.

- Smaller emittance, $\varepsilon_{n}=500 \mathrm{~m} \mathrm{~mm}-\mathrm{mrad}\left(\sigma_{\theta}=1.2\right)$, concentrates the energy in the foam and the interface temperature gradient is sharper but the tamper heating near the interface is higher.

- Larger emittance, $\varepsilon_{n}=1500 \mathrm{~m} \mathrm{~mm}$-mrad $\left(\sigma_{\theta}=3.7\right.$ degrees) spreads the energy. The average foam temperature and tamper temperatures are lower but there is a more gradual interface temperature gradient.

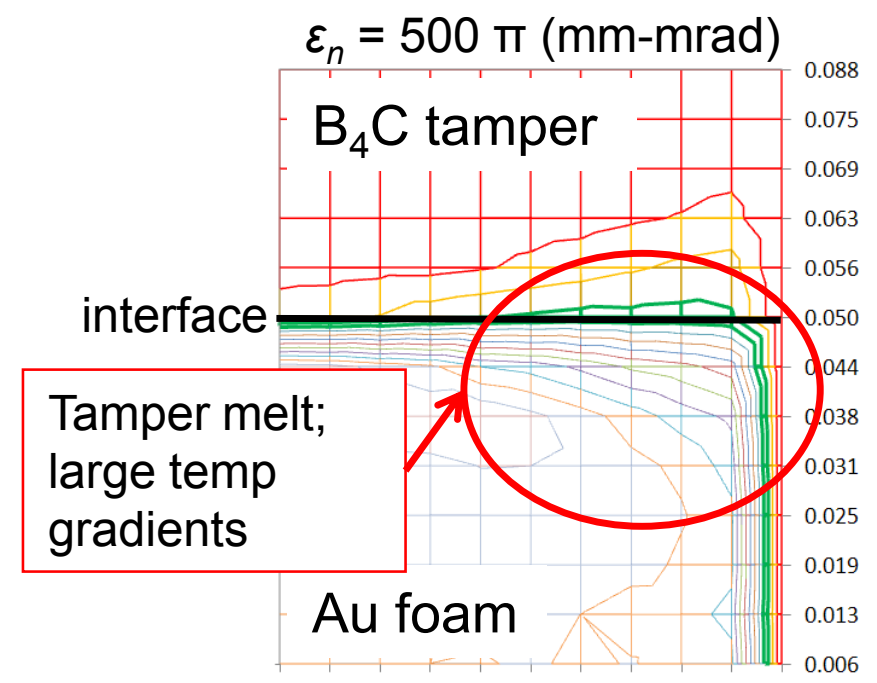

$\begin{array}{lllllllllll}0 & 0.01 & 0.02 & 0.03 & 0.04 & 0.05 & 0.06 & 0.07 & 0.08 & 0.09 & 0.1\end{array}$ $Z(\mathrm{~cm})$

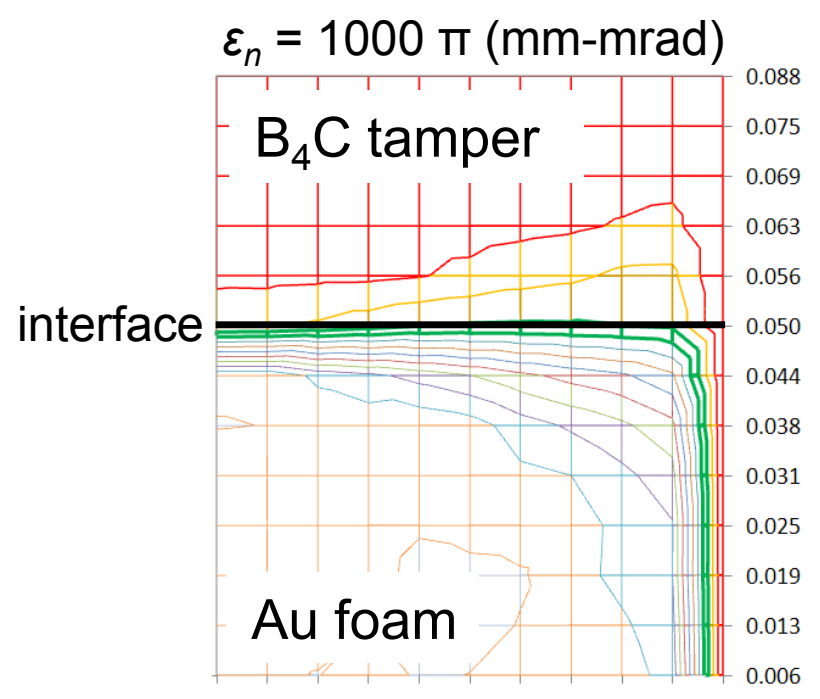

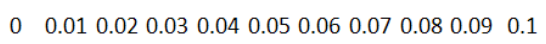
$\mathrm{Z}(\mathrm{cm})$

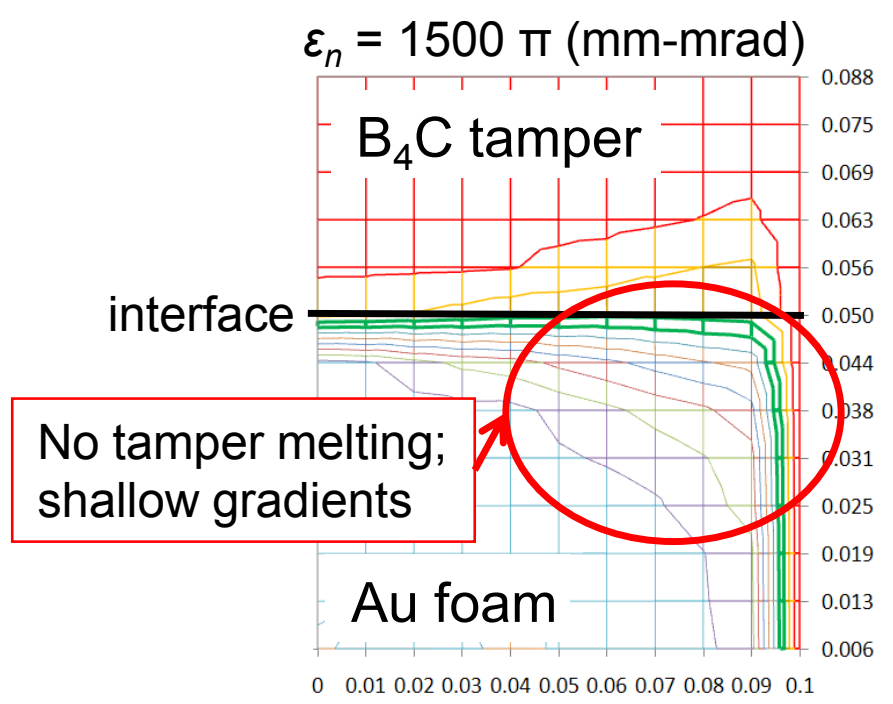
Z (cm) 


\section{Scattering electrons and interface gradients}

Energy-density gradients at the foam-tamper interface are due to unbalanced scattering.
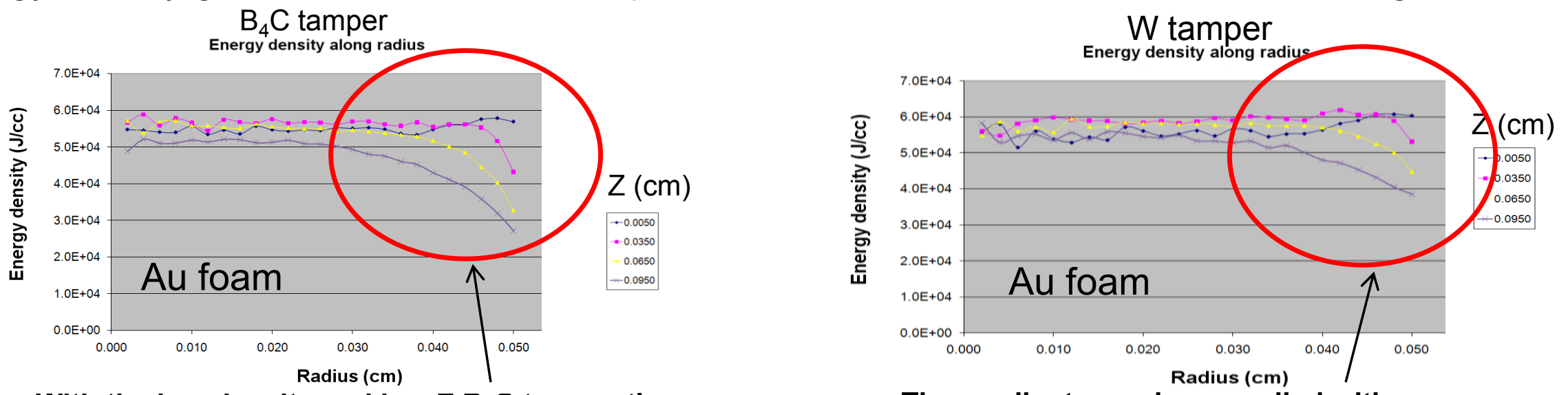

With the low density and low $Z \mathrm{~B}_{4} \mathrm{C}$ tamper the gradients in the Au foam are relatively large

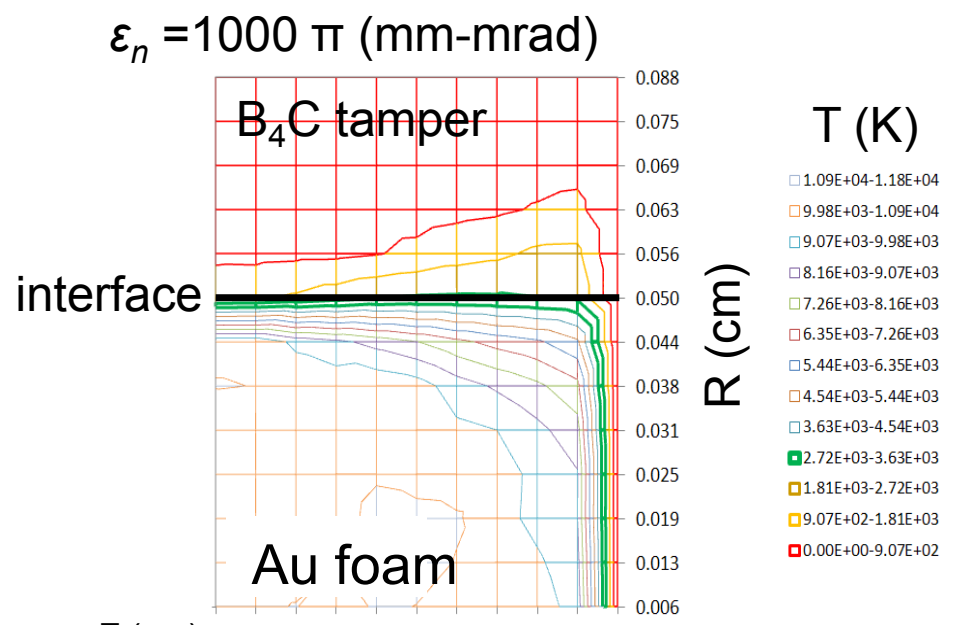

The gradients can be remedied with a high-density, high-Z W tamper

$$
\varepsilon_{n}=1000 \pi(\mathrm{mm}-\mathrm{mrad})
$$

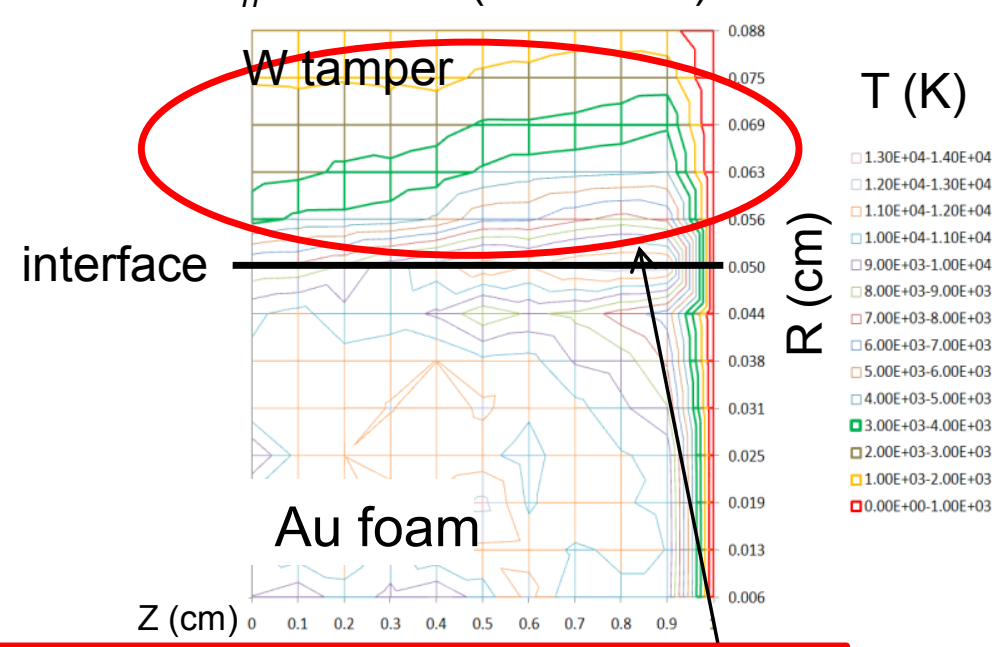

Unfortunately, while $\mathrm{B}_{4} \mathrm{C}$ heats but remains solid, a $\mathrm{W}$ tamper would melt (green band).

More work needs to be done on engineering the foam tamper interface 


\section{MCNP results used for an energy source in LASNEX}

LASNEX calculates the hydrodynamic evolution of material parameters (pressure, density, temp) of the foam-tamper target. In LASNEX, the energy source can be input instantaneously or ramped linearly over time. This option is useful for determining the sensitivity of a model to time-dependent conditions.

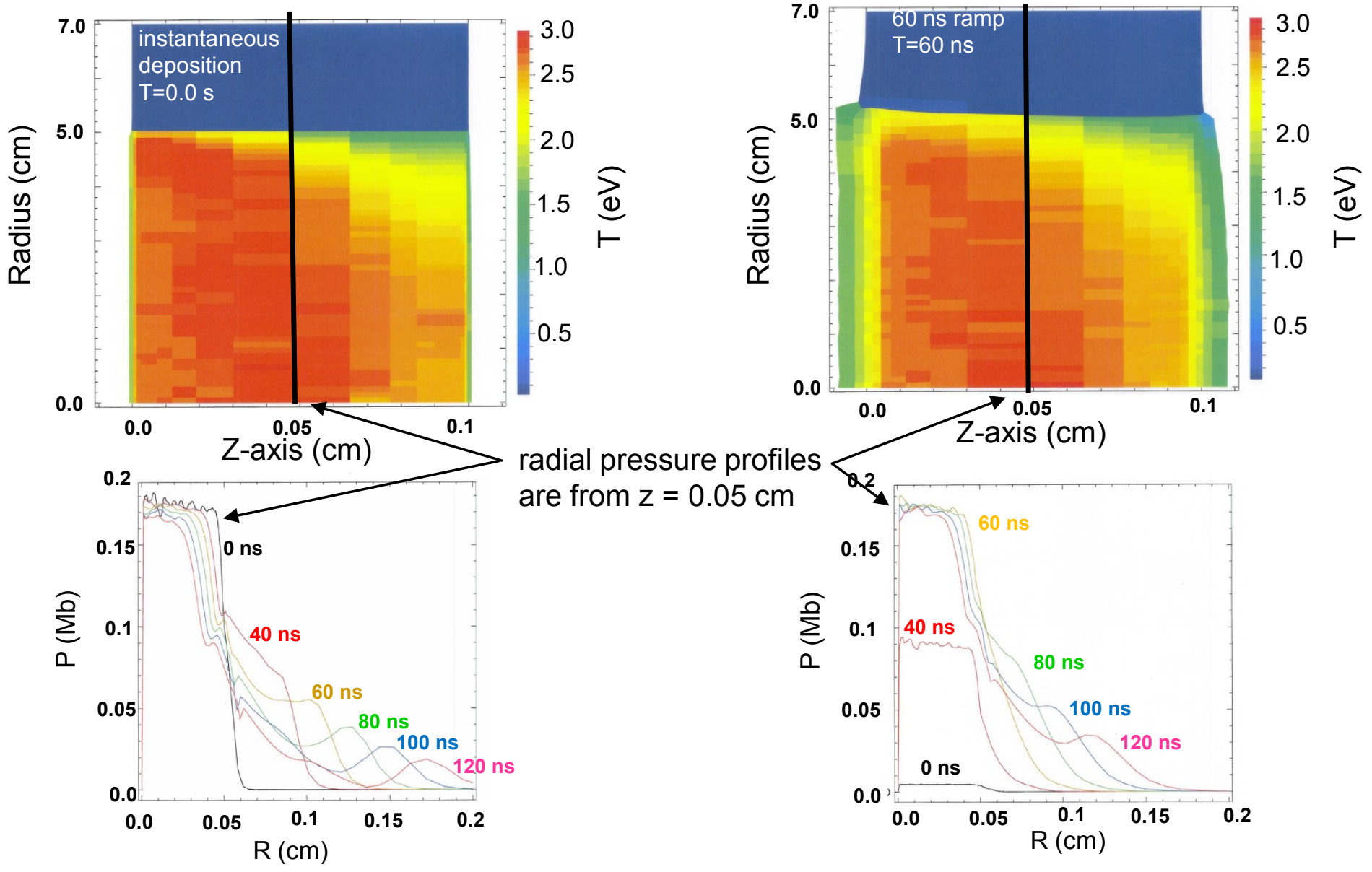

- Temperatures and pressures have similar magnitudes

- In the ramped case, edge effects and material motion are more pronounced

- Peak temperature slightly lower

- Pressure-wave amplitudes and "edges" are softer 


\section{LASNEX calculations show that a $6.4 \mathrm{~g} / \mathrm{cc}$ density Au foam tamped with $\mathrm{B}_{4} \mathrm{C}$ can be heated to a WDM temperature of $\sim 3 \mathrm{eV}$ with excellent radial uniformity}
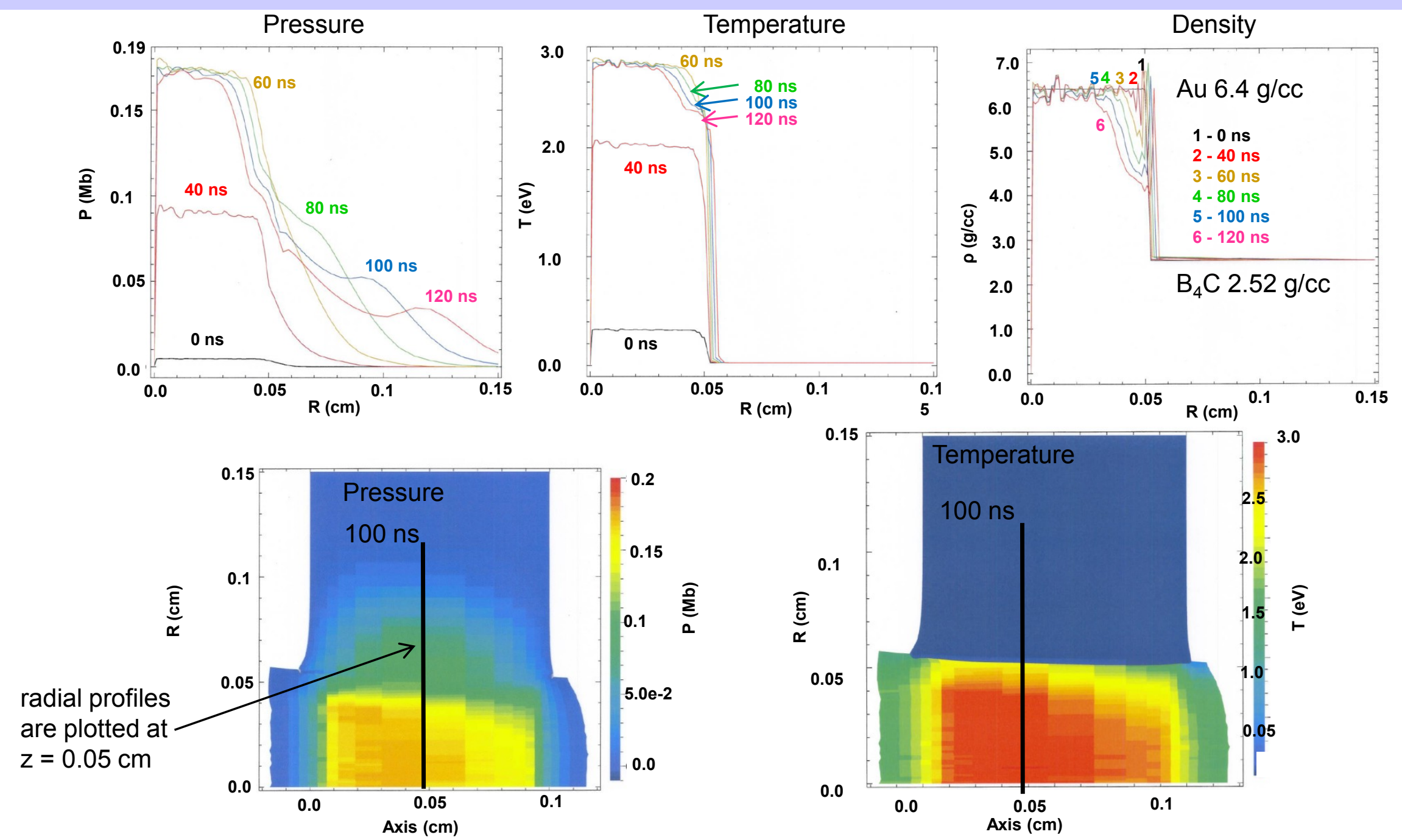

The pressure shockwave in the tamper travels outward with a speed of $\sim 10 \mathrm{~km} / \mathrm{s}$. 


\section{The shock wave in the tamper is related to temperatures and pressures in the foam}
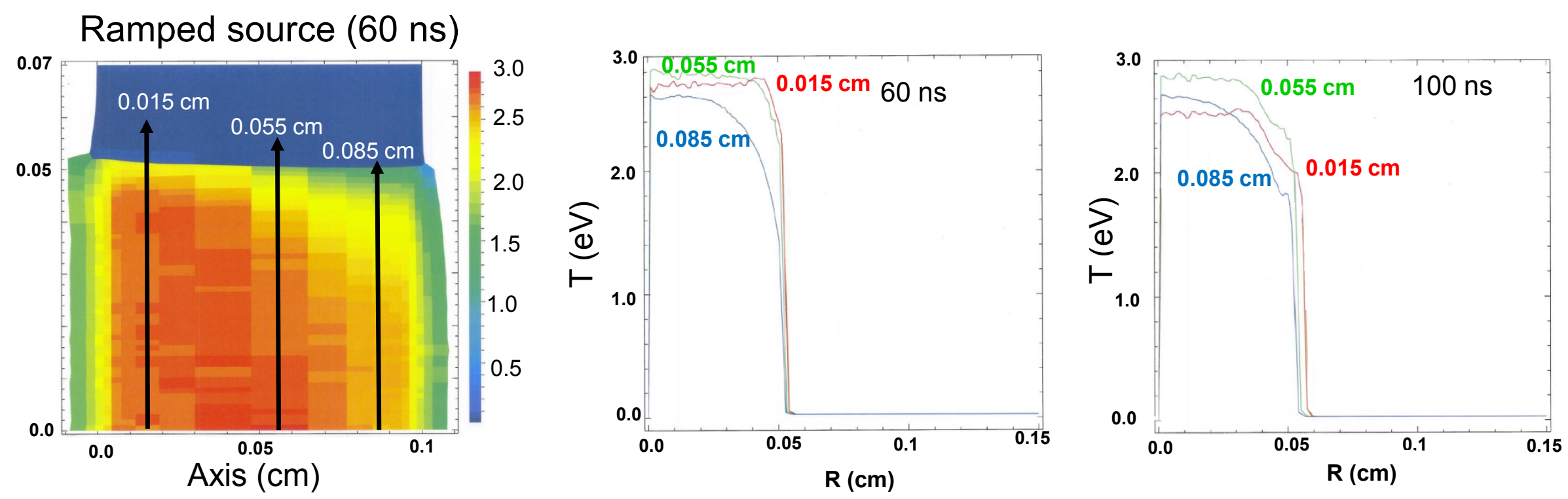

Radial profiles of the pressures and temperatures at three different points along the target axis and at two times show that the magnitude of the shock pressure depends upon with the temperature of the foam.
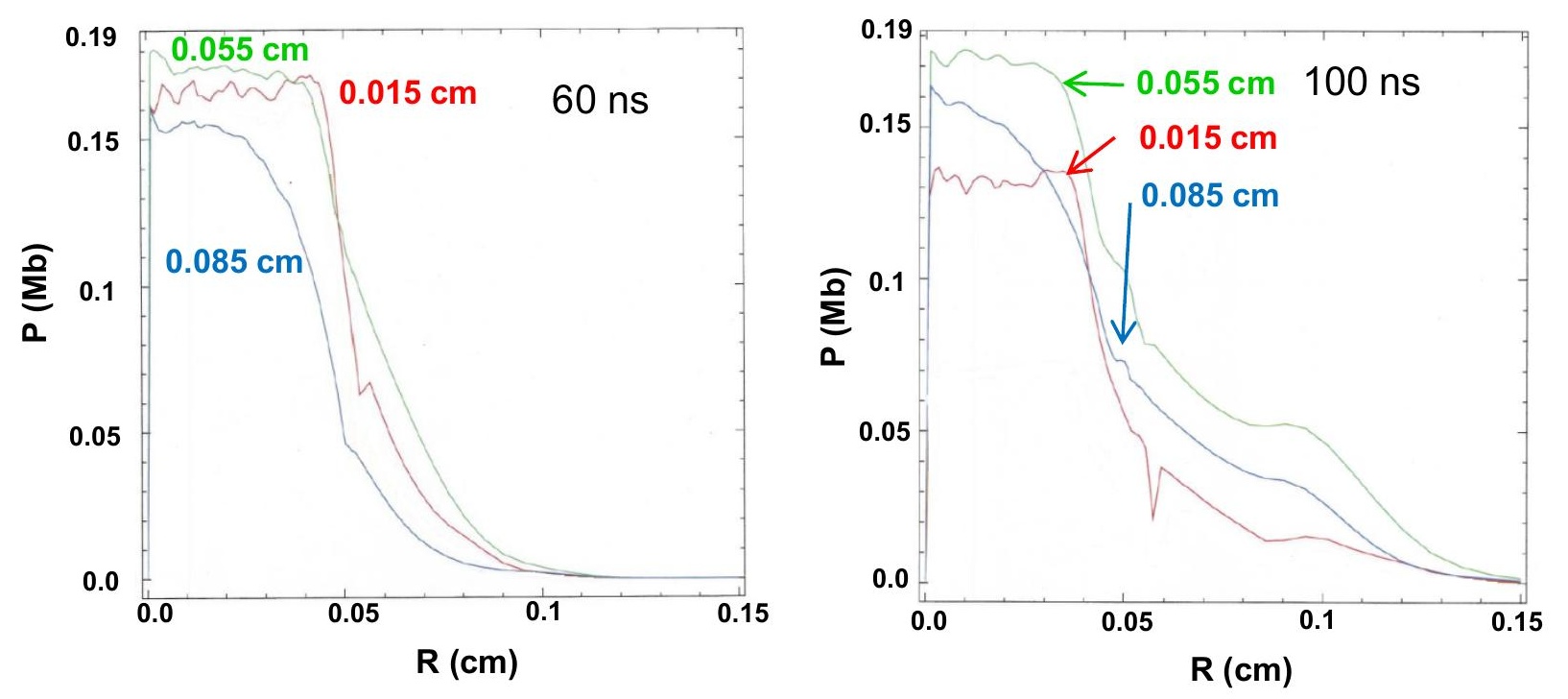


\section{A shockwave approaches the outer surface of the tamper at $120 \mathrm{~ns}$ with a $35 \mathrm{kBar}$ pressure and a velocity of $10.9 \mathrm{~km} / \mathrm{s}$}

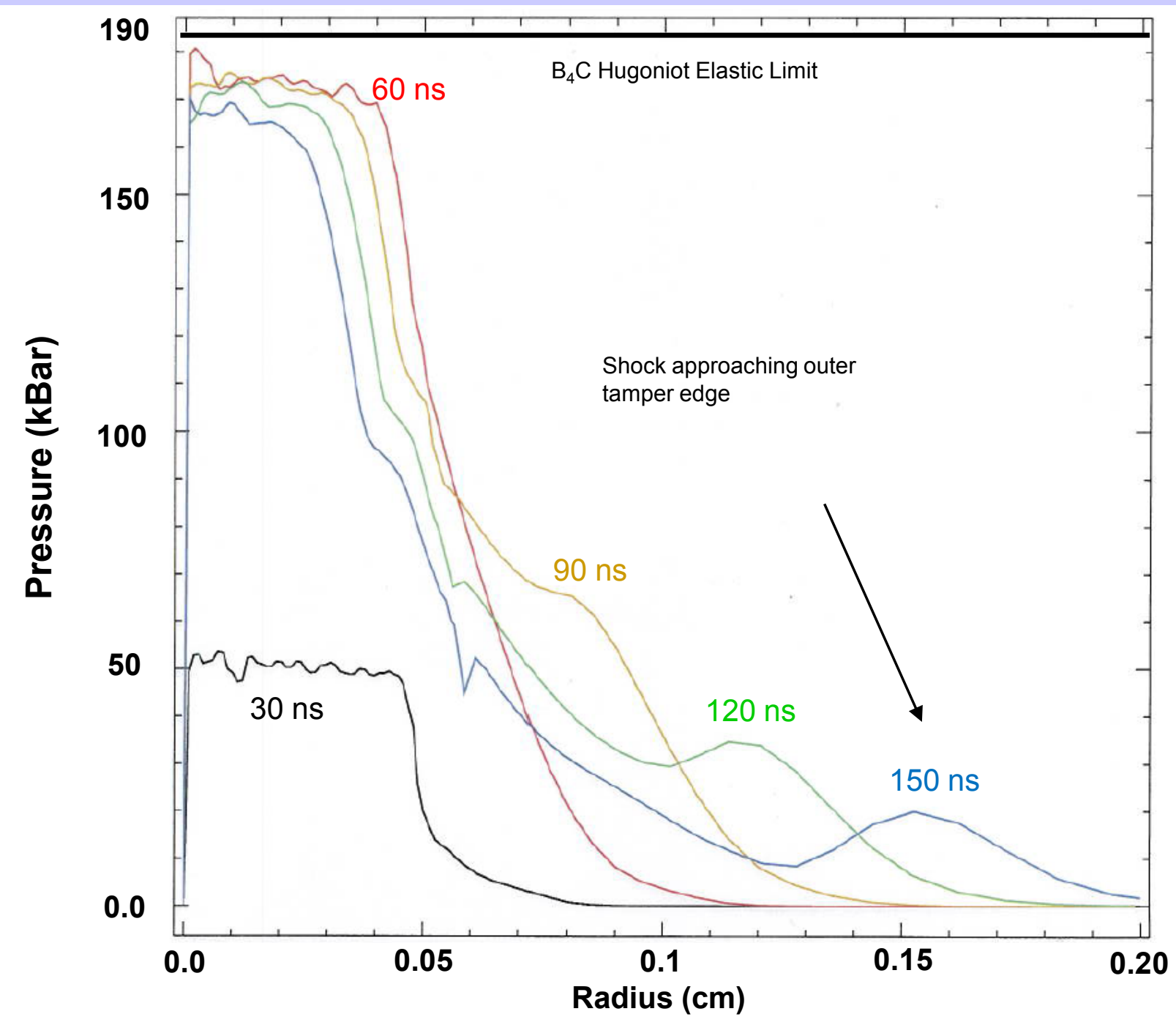

Radial line-outs at $z=0.05 \mathrm{~cm}$

Shock approaching outer

tamper edge

The shock pressure in the $B_{4} C$ is below the Hugoniot elastic limit (HEL) of 180 to 200 kBar. 


\section{PDV diagnostic will be used to measure the surface and shock velocities}

Diagnostic consists of

1. A stepped surface on the tamper

2. PDV diagnostic to

- measure the absolute velocity of the surface as the shock breaks out

Gold foam cylinder inside metal tamper - end view

- measure shock velocity from the difference between the breakout times at various steps

3. The measurements will infer the pressure in the gold WDM
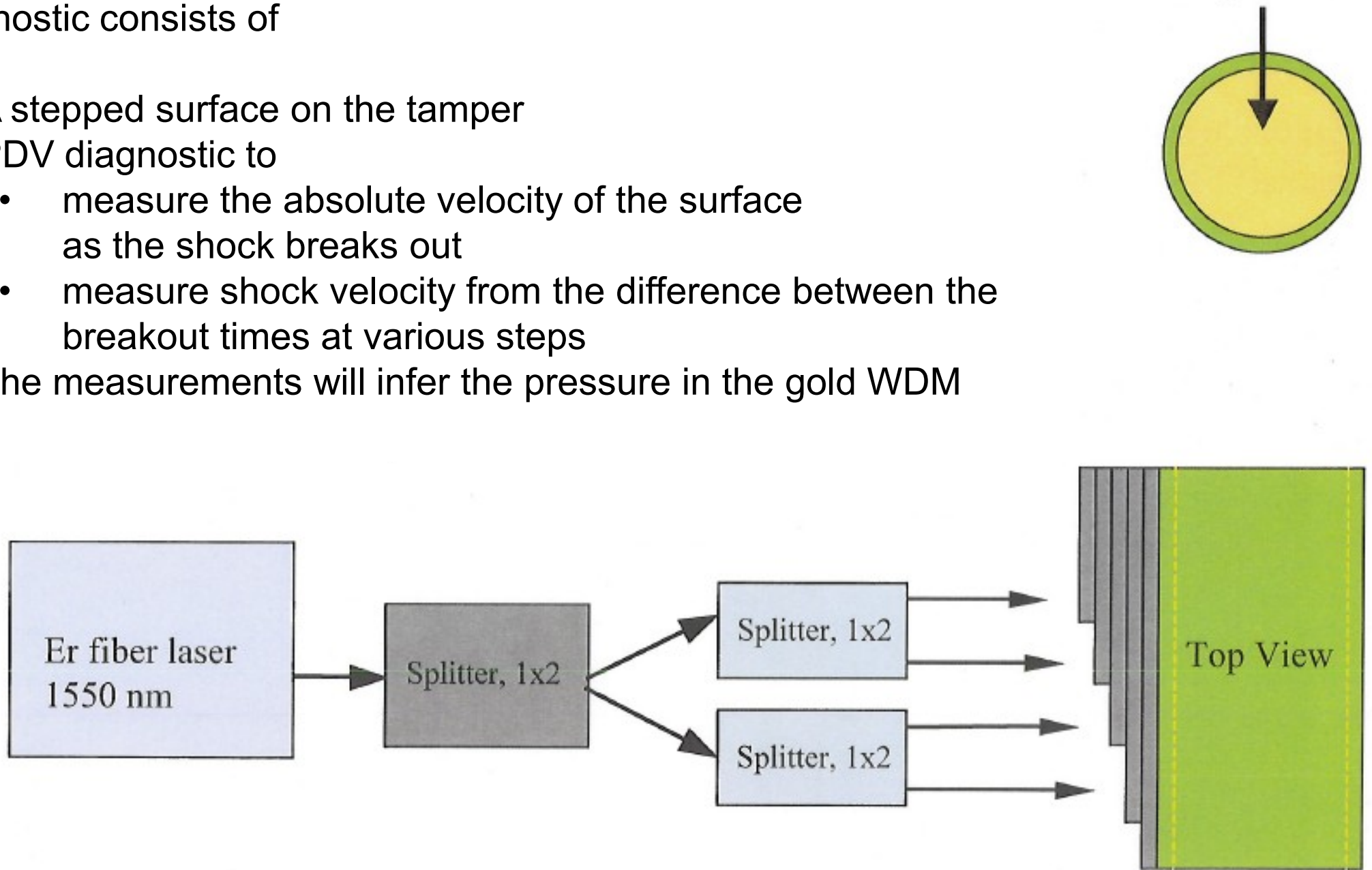


\section{A $10 \%$ difference in Au foam EOS pressure produces a measureable difference in shock breakout properties at $r=0.09 \mathrm{~cm}$}
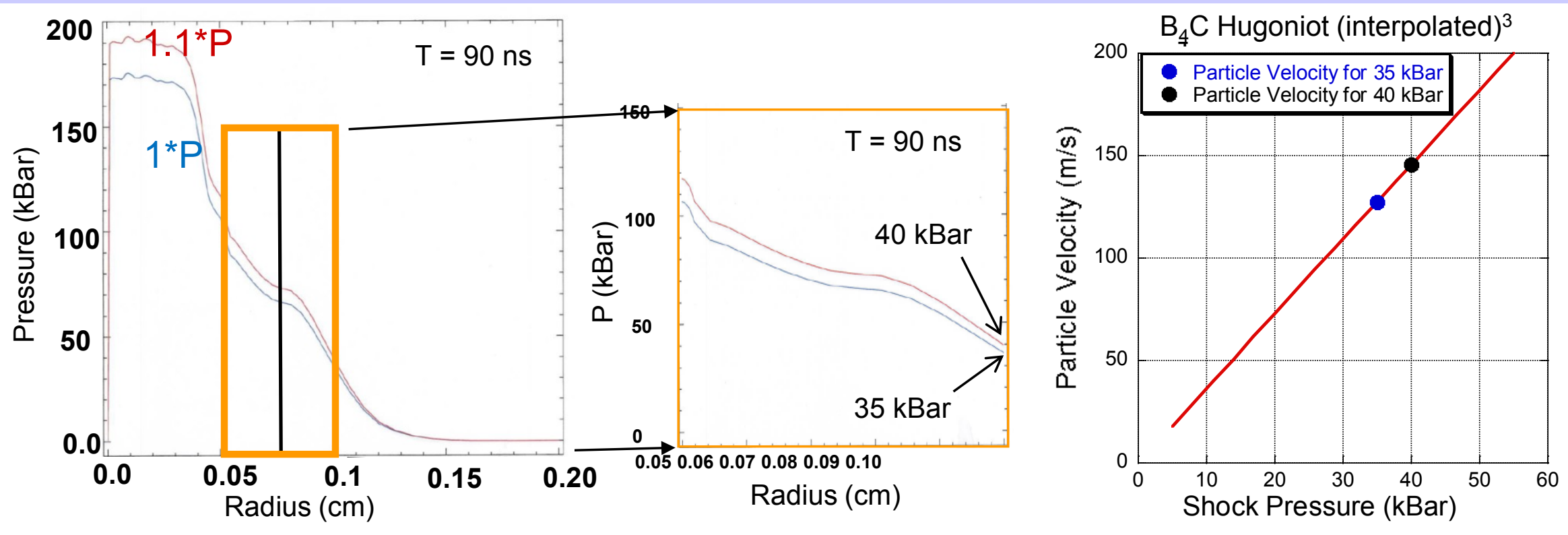

- Particle velocities can be resolved by standard velocimetry (PDV, VISAR) techniques to better than a few percent, which means pressure differences can be resolved to about $1 \mathrm{kBar}$.

- In our simulation, if the outer surface of the tamper were at $0.1 \mathrm{~cm}$ and $\mathrm{T}=90 \mathrm{~ns}, \mathrm{a} 10 \%$ difference in the shock pressure amplitude at the surface would produce release pressures $35 \mathrm{kBar}$ and $40 \mathrm{kBar}$. The $\mathrm{B}_{4} \mathrm{C}$ Hugoniot shows these pressures produce particle velocities of $128 \mathrm{~m} / \mathrm{s}$ and $146 \mathrm{~m} / \mathrm{s}$, respectively, a $15 \%$ difference that can easily be resolved with PDV (or VISAR).

- A $0.002 \mathrm{~cm}$ spatial separation at $10 \mathrm{~km} / \mathrm{s}$ gives a $2 \mathrm{~ns}$ breakout difference. This time difference can be resolved to high precision with a state-of-the-art PDV ( $\sim 0.25 \mathrm{~ns}$ resolution) system.

- Error in particle velocity measurements should diminish as pressures approach the acoustic limit.

3Dandekar, Dattatraya P., "Shock Response of Boron Carbide," Army Research Laboratory, ARL-TR-2456 (April 2001). 


\section{The DARHT electron spectrometer determines the energy deposited into the Au foam to high accuracy}

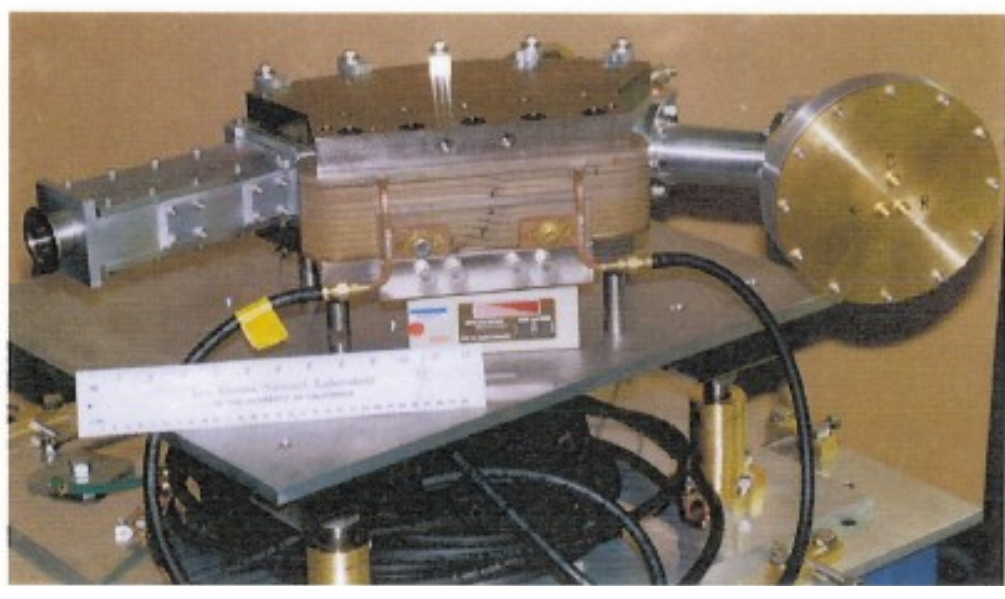

Spectrometer shown with calibration detectors
18.0
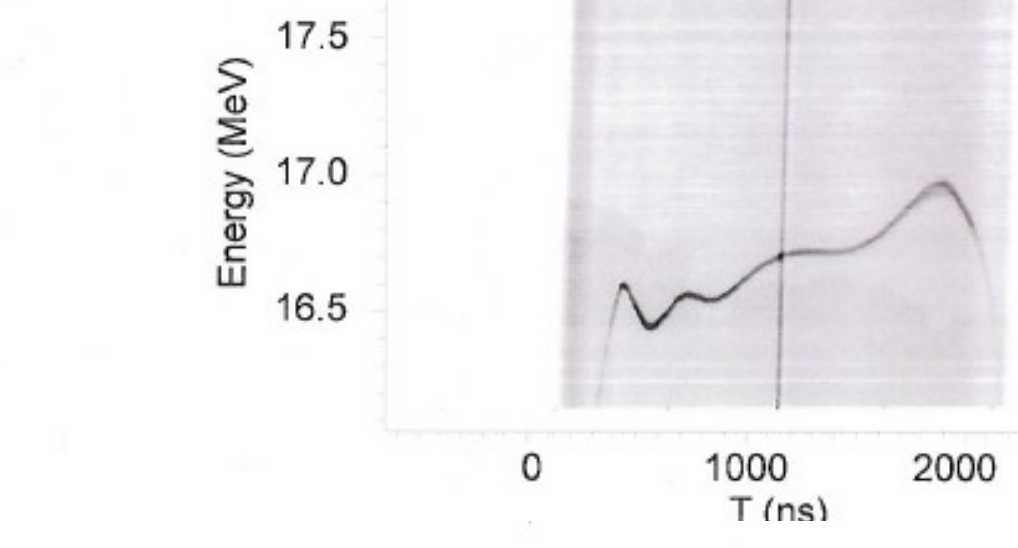

Electron energy range: $2.5 \mathrm{MeV}-34 \mathrm{MeV}$

Detection window: $\sim \pm 5 \%$

Absolute accuracy: $\pm 1 \%$ (calibrated with purpose-built ion accelerator)

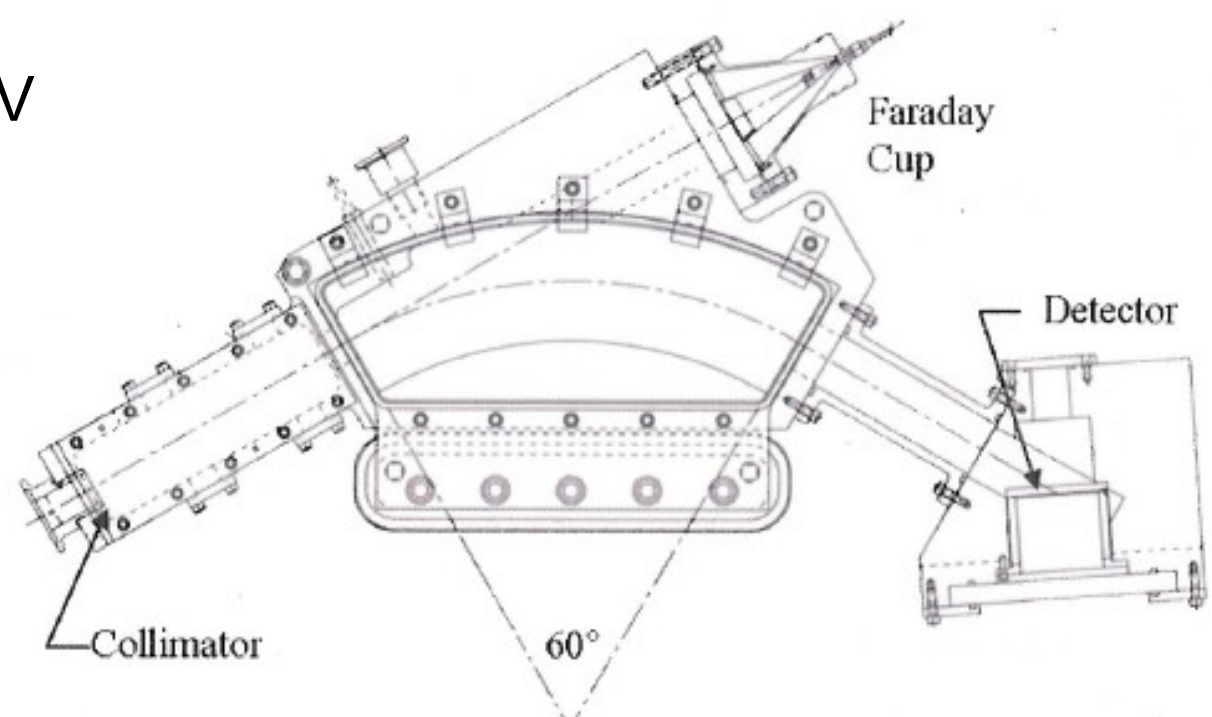




\section{Summary}

- MCNP and LASNEX calculations have been perform to determine if WDM targets can be achieved on DARHT.

- Cell-by-cell time-dependent energy deposition from the 60 ns DARHT pulse was approximated with a linear ramp.

- LASNEX indicates that target temperatures in the few eV regime can be achieved.

- A velocimetry technique to measure the EOS pressure generated in a $\mathrm{B}_{4} \mathrm{C}$ tamper has been proposed.

- EOS pressures measurements are expected to be measured with $10 \%$ or better accuracy. 